\title{
SOLUÇÕES PARA SUBSTITUIÇÃO DOS PLCS DE UMA PLANTA DE SINTERIZAÇÃO*
}

\author{
Marcos Velho Ludolf ${ }^{1}$ \\ Gabriel Nazareth Guedes Alcoforado²
}

\section{Resumo}

O projeto de Substituição dos Controladores Lógicos Programáveis da Sinterização tem como objetivo a substituição dos PLCs da Família S5 (Siemens) já obsoletos por novos PLCs de tecnologia atual. O projeto de substituição foi dividido em etapas, sendo que a primeira etapa ocorreu em 2012. Consequentemente, teremos novos e antigos PLCs na mesma plataforma de controle até o fim do projeto. Os maiores desafios foram fazer toda a migração e adaptação de hardware e software dos novos PLCs ao restante da planta adequando-se aos sistemas existentes tais como PIMS, comunicação com o Nível 2, comunicação com balanças já obsoletas, SCADA, outros PLCs e concluir toda a migração da primeira etapa do projeto em apenas 60 dias compreendendo desde a desmontagem do sistema anterior, montagem dos painéis novos, recomissionar todo o $\mathrm{l} / \mathrm{O}$ substituído, conciliar todos os testes à frio e parciais com demais serviços de manutenção programados e realizar o comissionamento a quente de toda a sequência da planta nos últimos 04 dias de implantação, quando todas as linhas de operação foram liberadas para testes. Em 2014 foi iniciada uma segunda etapa, e um PLC de 600 pontos de I/O já foi substituído em apenas $80 \mathrm{~h}$ usando uma nova estratégia.

Palavras-chave: Sinterização; Siemens S5; PLC.

\section{Abstract}

\section{SOLUTIONS FOR SINTER PLANT PLC EXCHANGE}

The draft Replacement of Programmable Logic Controllers in Sintering aims to replace the S5 PLCs Family (Siemens) already obsolete by new PLCs current technology. The replacement project was divided into stages, with the first stage occurred in 2012. Consequently, we will have new and old PLCs on the same platform control until the end of the project. The biggest challenges were to do all the migration and adaptation of hardware and software of the new PLCs to the rest of the plant adapting to existing systems such as PIMS, communication with the Level 2 communication with deprecated scales, SCADA, PLCs and complete all other the migration of the first stage of the project in just 60 days, ranging from the removal of the previous system and installation of new panels, recomissionar all I/O replaced, combine all the cold and partial tests with other scheduled maintenance services and perform commissioning hot of the entire sequence of the plant in the last 04 days of implantation, when all lines of operation have been released for testing. In 2014 started a second step, and a PLC 600 points of $\mathrm{I} / \mathrm{O}$ has been replaced in just $80 \mathrm{~h}$ using a new strategy.

Keywords: Sinter Plant; Siemens S5; PLC.

1 Mestre em Eng. Elétrica, Especialista em Projeto Eletroeletrônico, Divisão de Engenharia de Manutenção, ArcelorMittal Tubarão, Serra, ES, Brasil.

2 Engenheiro Elétricista, Especialista em Projeto Eletroeletrônico, Divisão de Engenharia de Manutenção, ArcelorMittal Tubarão, Serra, ES, Brasil.

* Contribuição técnica ao $18^{\circ}$ Seminário de Automação e TI Industrial, 23 a 26 de setembro de 2014, São Paulo, SP, Brasil. 


\section{INTRODUÇÃO}

A metalurgia é o conjunto de técnicas que permitem a extração, a manipulação de metais e a geração de ligas metálicas. A siderurgia é o ramo da metalurgia que se dedica à fabricação e tratamento de aços e ferros fundidos. O aço é produzido, basicamente, a partir de minério de ferro, carvão e cal. A fabricação do aço pode ser dividida em cinco etapas: preparação da carga, redução, refino, lingotamento e laminação [1], conforme mostra Figura 1.

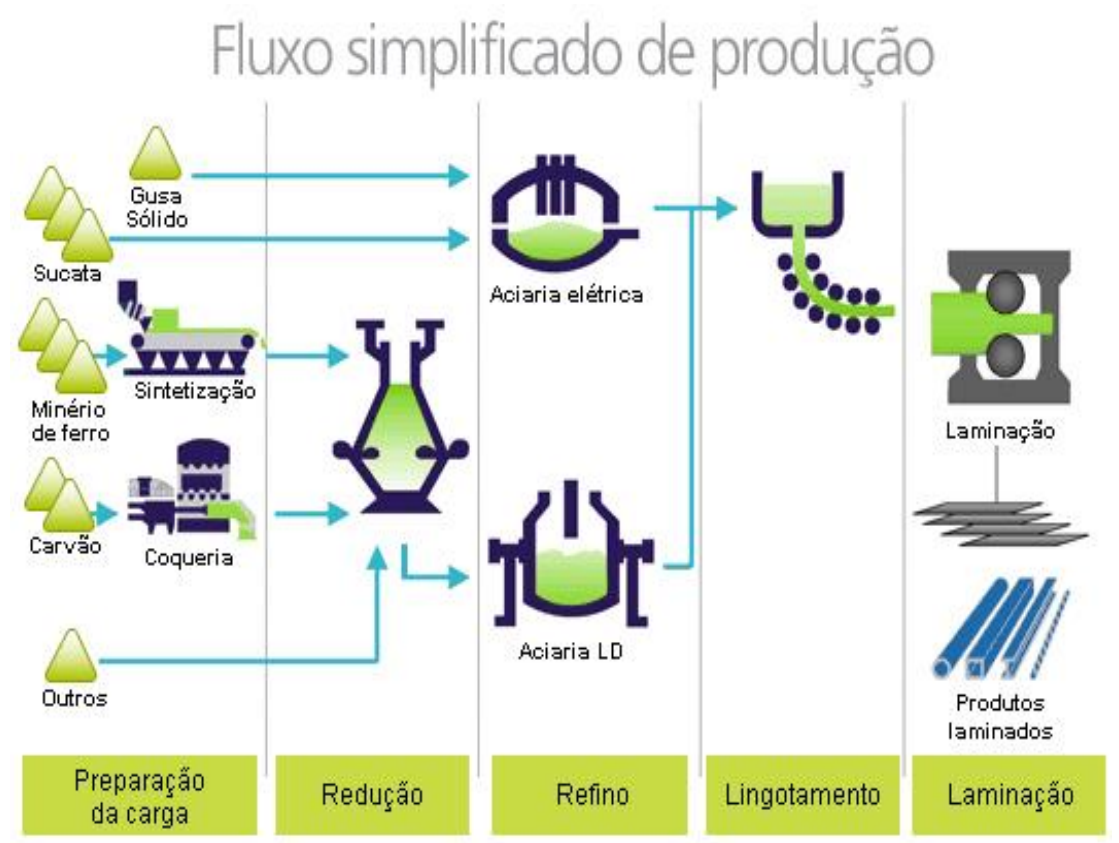

Figura 1. Fluxo simplificado de produção do aço (Instituto Aço Brasil, 2009).

Durante a preparação da carga, grande parte do minério de ferro (finos) é aglomerada, resultando em um produto chamado "sinter". O carvão processado na Coqueria se transforma em "coque". O sinter e o coque são carregados ao altoforno, onde o coque, em contato com o oxigênio, produz calor que funde a carga metálica e dá início ao processo de redução do minério de ferro em um metal líquido: o ferro-gusa. No refino, as aciarias são utilizadas para transformar o gusa e a sucata (resíduos descartados) em aço líquido. A maior parte do aço líquido é solidificada em equipamentos de lingotamento contínuo para produzir semiacabados, lingotes e blocos. Os produtos resultantes do lingotamento são processados por laminadores e transformados em uma grande variedade de produtos siderúrgicos.

O processo de sinterização, isto é, de aglomeração de partículas, é crucial para o êxito da produção de aço via alto-forno, dada às limitações que este tipo de reator apresenta em relação à presença de material fino [2]. A elevada quantidade de material fino no alto-forno reduz a permeabilidade do gás dentro do reator, a velocidade das reações de redução e a velocidade de produção do gusa. As matérias-primas utilizadas são: minério, lama, coque e finos de minério.

A mistura das matérias primas segue numa correia transportadora, formando o "blend" ou mistura de sinterização. O blend é misturado em um tambor rotativo, chamado de tambor de mistura, de forma a se obter uma mistura homogênea das matérias-primas. Em seguida, ele segue por um silo até chegar ao forno de ignição, onde a mistura homogênea é acessa. A máquina de sinter funciona como um

* Contribuição técnica ao $18^{\circ}$ Seminário de Automação e TI Industrial, 23 a 26 de setembro de 2014, São Paulo, SP, Brasil. 
cigarro, depois de acesa, as caixas de vento localizadas abaixo da esteira mantém a queima do material. Durante a ignição, a temperatura na parte superior da camada de sinter varia entre $1200^{\circ} \mathrm{C}$ a $1400^{\circ} \mathrm{C}$. Após um período curto de ignição, o calor do topo da mistura de sinterização inicia o movimento descendente ao longo da camada de mistura. Antes da camada da zona de combustão, ocorre a evaporação da água e componentes voláteis, removendo as impurezas prejudiciais a partir do minério, como o enxofre e fósforo. Já na zona de combustão, área final de queima, ocorrem as reações de aglomeração entre as partículas da mistura e aglomerados resistentes são formados. A maioria do calor presente na zona de combustão é absorvida pela secagem, calcinação e aquecimento das camadas inferiores do leito. Quando a zona de combustão atinge o fundo do leito da mistura de sinterização, o processo é finalizado. O sinter obtido é grosseiramente quebrado por um britador. Após o resfriamento, o sinter é peneirado e o restante é enviado para os silos do alto-forno.

A sinterização da ArcelorMittal Tubarão iniciou o processo de digitalização do sistema de controle da planta em 1997, quando foram instalados PLCs Siemens S5 em todos os sub-processos, existentes na época. Em 2007 houve a expansão da capacidade da usina de 5,0 para 7,5 milhões de toneladas/ano de aço, foram acrescentados novos equipamentos na área da Sinterização. Para executar o controle destes, novos PLCs Siemens, agora da família S7, foram instalados.

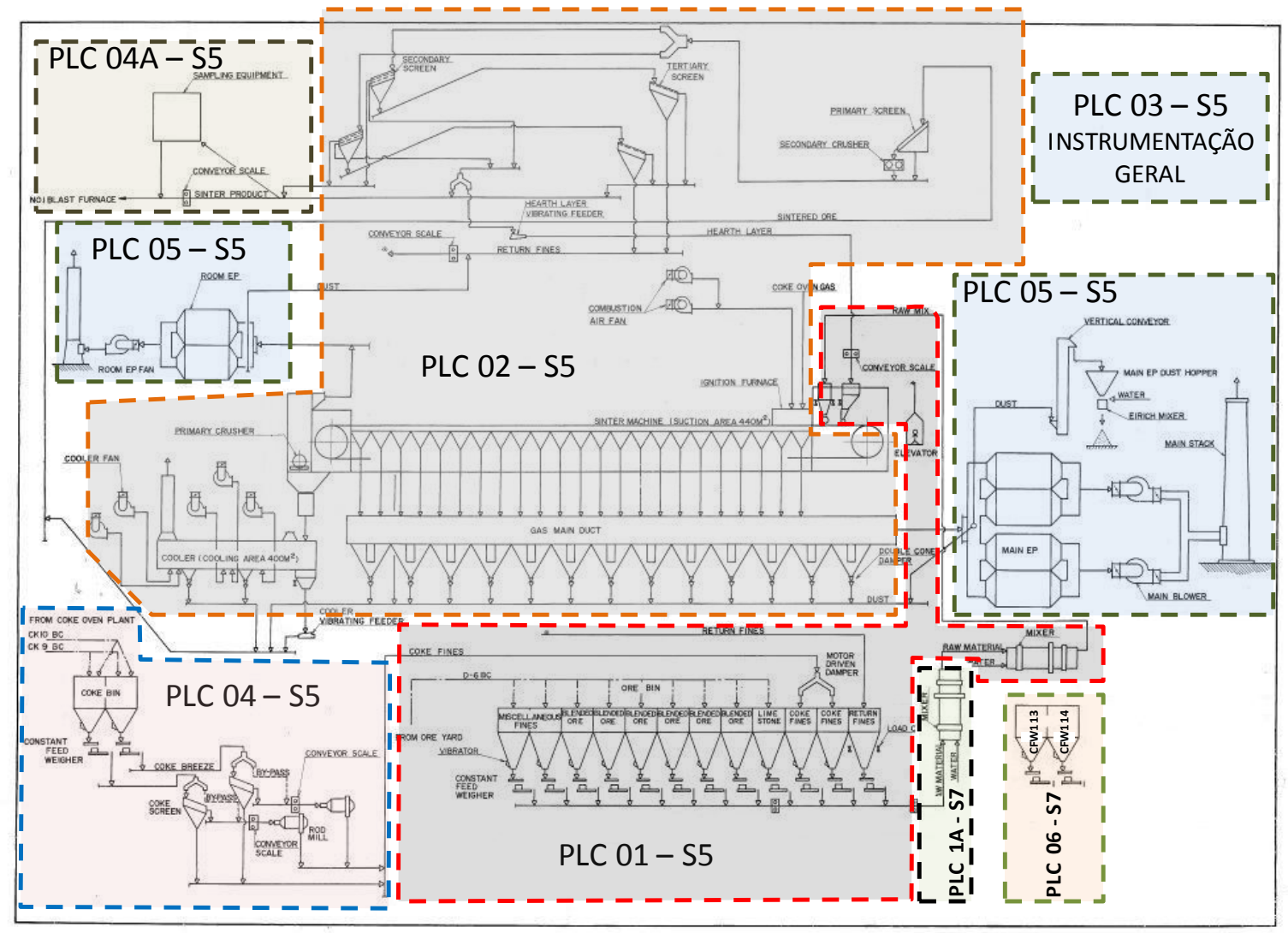

Figura 2. Configuração dos PLCs da Sinterização em 2007.

A configuração final em 2007 permaneceu até 2012. A divisão de funcionalidade de cada PLC, como mostra a figura 2 era:

- PLC 1 - Linha de Mistura e Nodulizador;

- PLC 1A - Misturador;

* Contribuição técnica ao $18^{\circ}$ Seminário de Automação e TI Industrial, 23 a 26 de setembro de 2014, São Paulo, SP, Brasil. 
- PLC 2 - Máquina de Sinter, Resfriador, Linha de Sinter Produto e Retorno de Finos;

- PLC 3 - Instrumentação Geral da Planta;

- PLC 4 - Moagem de Coque;

- PLC 4A - Amostragem de Sinter;

- PLC 5 - Precipitadores Eletroestáticos (EP) Primário e Secundário;

- PLC 6 - Adição de Cal e Pó de EP;

Em 2005 o PLC Siemens S5 entrou em fase de descontinuação, que será concluída em 2015. Porém para realizar a substituição de todos os PLCs S5 da Sinterização haveria a necessidade de uma parada de duração muito superior às paradas de manutenção programada, caso contrário o funcionamento dos Altos Fornos seria diretamente prejudicado. Podemos avaliar a dimensão desta tarefa na tabela 01 , onde temos a quantidade de I/Os de cada PLC.

Tabela 1. Quantidade de I/Os dos PLCs da Sinterização

\begin{tabular}{|l|r|r|r|r|r|r|r|r|r|}
\hline PLC & 1 & 2 & 3 & 4 & $4 A$ & Total S5 & \multicolumn{1}{|c|}{1 A } & 6 & Total S7 \\
\hline & \multicolumn{3}{|c|}{ Siemes S5 } & & & \multicolumn{2}{|c|}{ Siemens S7 } & \\
\hline DI & 496 & 1024 & 80 & 656 & 496 & $\mathbf{2 7 5 2}$ & 160 & 128 & $\mathbf{2 8 8}$ \\
\hline DO & 112 & 240 & 32 & 144 & 128 & $\mathbf{6 5 6}$ & 32 & 48 & $\mathbf{8 0}$ \\
\hline Al & 40 & 40 & 112 & 8 & 8 & $\mathbf{2 0 8}$ & 16 & 8 & $\mathbf{2 4}$ \\
\hline AO & 8 & 8 & 24 & 8 & 8 & $\mathbf{5 6}$ & 4 & 0 & $\mathbf{4}$ \\
\hline \hline Total & 656 & 1312 & 248 & 816 & 640 & 3672 & 212 & 184 & 396 \\
\hline
\end{tabular}

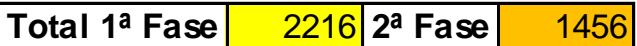

Em 2012, em função da parada para reforma do Alto Forno 01 da AMT, houve a oportunidade de parar totalmente a Sinterização por um período maior que 30 dias. Durante esta parada também seria realizada a substituição do Resfriador de Sinter, realizada pela empresa Paul Wurth. Esta parada deveria ser aproveitada, pois não se repetirá em menos de 10 a 15 anos. Portanto foram avaliadas estrategicamente as possibilidades e decidido realizar esta substituição em duas etapas: a primeira consistiria da substituição dos PLCs 1, 2 e 3; enquanto que na segunda haveria a troca dos PLCs 4 e 4A. O PLC 5 não seria trocado neste projeto em função de uma provável modernização no equipamento nos próximos anos.

Foi realizada uma concorrência e contratada a empresa TSA para realizar este serviço. As atividades começaram em Janeiro de 2012, o que aumentou o desafio uma vez que a parada estava prevista para Maio/Junho do mesmo ano.

\section{MATERIAIS E MÉTODOS}

Neste capítulo serão descritas as duas fases do projeto, iniciando-se pela executada em 2012.

\section{$2.11^{\text {a }}$ Etapa de Migração - 2012}

Além do desafio intrínseco de migrar 2.216 pontos, havia as restrições do prazo da engenharia, fornecimento, desenvolvimento e teste do software (05 meses); e do prazo de montagem, testes e start-up (35 dias).

Dividimos as atenções em 02 focos principais: hardware (estrutura dos PLCs e painéis) e software (arquitetura do software).

\footnotetext{
* Contribuição técnica ao $18^{\circ}$ Seminário de Automação e TI Industrial, 23 a 26 de setembro de 2014, São Paulo, SP, Brasil.
} 


\subsubsection{Hardware}

Como a principal motivação do projeto era a substituição do hardware descontinuado, deveríamos substituir todo o PLC, mas de forma mais rápida possível. Nesta migração também foi incluída a instalação de seletividade da alimentação de controle dos equipamentos, anteriormente dividida por sub-processo. A interligação dos PLCs com o campo na Sinterização da AMT passa por dois painéis intermediários: ARP e TP. Nos ARPs existem relés para o acionamento de cargas no campo ou em CCMs, em quanto que os TPs são apenas painéis de bornes.

A solução foi trocar apenas os painéis dos PLCs, colocando os novos painéis sobre as bases dos antigos, aproveitando todos os cabos de interligação entre PLCs e ARPs (figura 03). Para facilitar a instalação, cada painel possuía toda distribuição interna de cada nível de tensão dos seus respectivos I/Os, eliminando a necessidade de passagem de cabos entre os novos painéis. A distribuição dos I/Os dentro das colunas e borneiras foram realizadas de forma a preservar a distribuição anterior. A nova seletividade de alimentação dos equipamentos foi realizada instalando-se disjuntores nos respectivos ARP's, atividade que poderia ser realizada em paralelo a instalação dos painéis dos PLCs, permitindo também uma alocação interna melhor distribuída. Todo o hardware utilizado nesta etapa foi da família S7-300 por apresentar o melhor custo e melhor aproveitamento do espaço dos painéis.

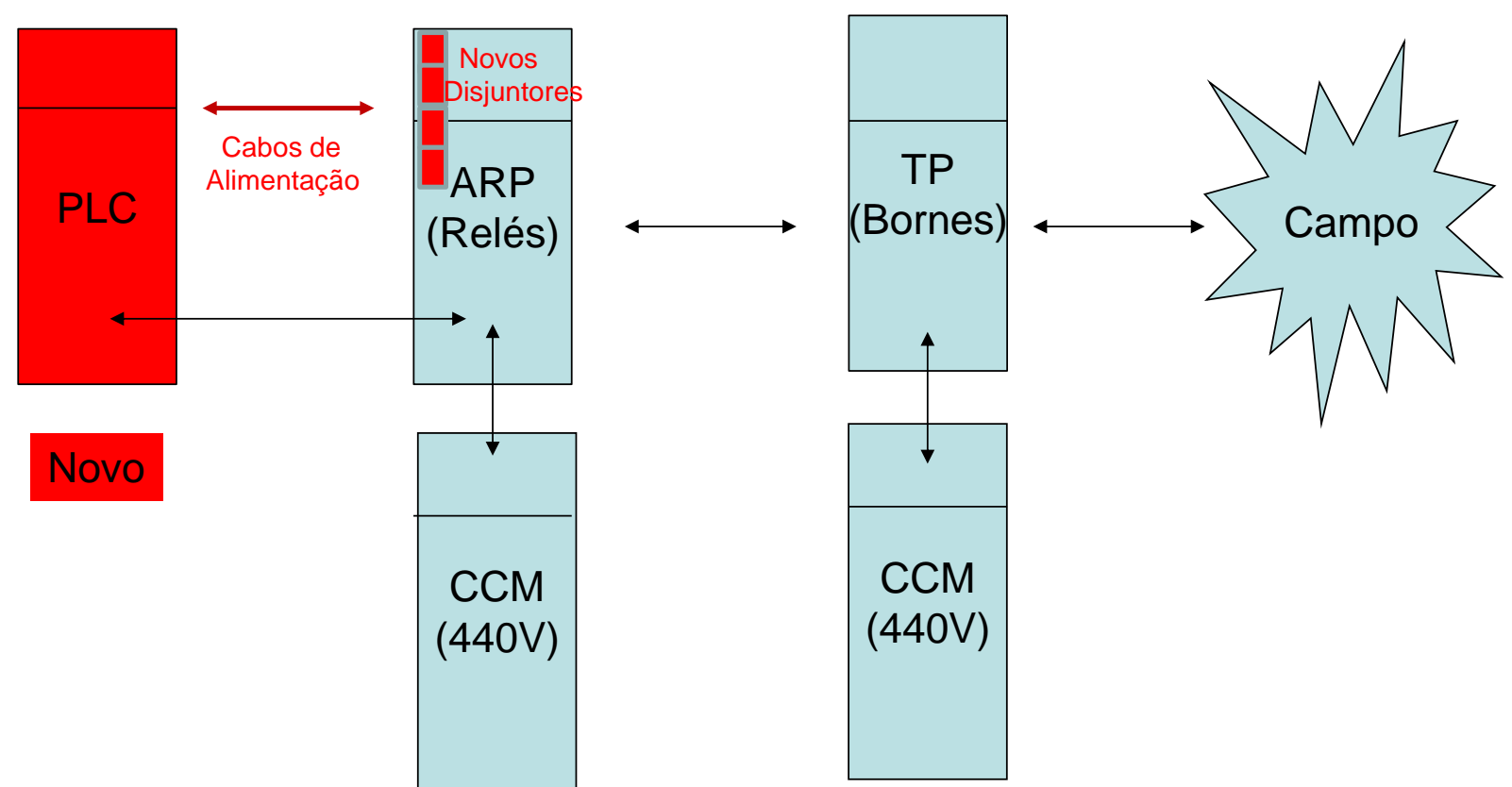

Figura 3. Configuração da interligação painéis de controle da Sinterização.

Foram 06 colunas de painel trocadas divididas da seguinte forma: 02 colunas do PLC 1, 03 colunas do PLC 2 e 01 Coluna do PLC 3.

\subsubsection{Software}

A migração do software deveria atender duas missões: manter as mesmas condições lógicas do processo, melhorar a interface entre os PLCs S5 e S7, e unificar o sistema supervisório (antes dividido em dois aplicativos: um realiza a interface com os PLCs 1, 2, 3 e 5; enquanto o outro com os PLCs 4 e 4A).

\footnotetext{
* Contribuição técnica ao $18^{\circ}$ Seminário de Automação e TI Industrial, 23 a 26 de setembro de 2014,
} São Paulo, SP, Brasil. 
Para facilitar a execução da lógica foram padronizadas as áreas de interface com o sistema supervisório desenvolvido no InTouch. Estas áreas compreendiam as seguintes informações de cada equipamento (quando aplicável): Estados, Defeitos, Comandos, Intertravamentos. Em cada respectiva área, havia uma palavra para cada equipamento com a mesma estrutura de dados, como podemos observar no exemplo da tabela 2.

Tabela 2. Palavra de Estados para Válvula Dupla / Damper

\begin{tabular}{|l|l|l|}
\hline \multicolumn{3}{|c|}{ Estados Válvula Dupla / Damper } \\
\hline Mnemônico & \multicolumn{1}{|c|}{ Função } & Bit \\
\hline EZAHA & Aberta & 0.0 \\
\hline & Reserva & 0.1 \\
\hline EZALF & Fechada & 0.2 \\
\hline EIMP & Impedido & 0.3 \\
\hline EPPP & Pronto Para Acionamento & 0.4 \\
\hline ELOC & Local Selecioando & 0.5 \\
\hline EREM & Remoto Selecioando & 0.6 \\
\hline & Reserva & 0.7 \\
\hline EMAN & Manual Selecionado & 1.0 \\
\hline E AUTO & Automático Selecioando & 1.1 \\
\hline EABRI & Abrindo & 1.2 \\
\hline EFECH & Fechando & 1.3 \\
\hline & Reserva & 1.4 \\
\hline EDL & Disjuntor Ligado & 1.5 \\
\hline EINT & Resumo de Intertravamentos & 1.6 \\
\hline EDEF & Resumo de Defeitos & 1.7 \\
\hline
\end{tabular}

Também foram criados blocos típicos, que executam funções recorrentes necessárias à lógica de funcionamentos dos diversos equipamentos. Estes blocos reduzem a possibilidade de erro pontual, facilitam a avaliação do funcionamento da lógica e agiliza a programação. Os blocos criados atenderam as seguintes funções: Partida Direta ou Reversa de Motores; Acionamento Simples de Válvula; Acionamento Duplo de Válvula; Tratamento de Variáveis Analógicas.

* Contribuição técnica ao $18^{\circ}$ Seminário de Automação e TI Industrial, 23 a 26 de setembro de 2014, São Paulo, SP, Brasil. 


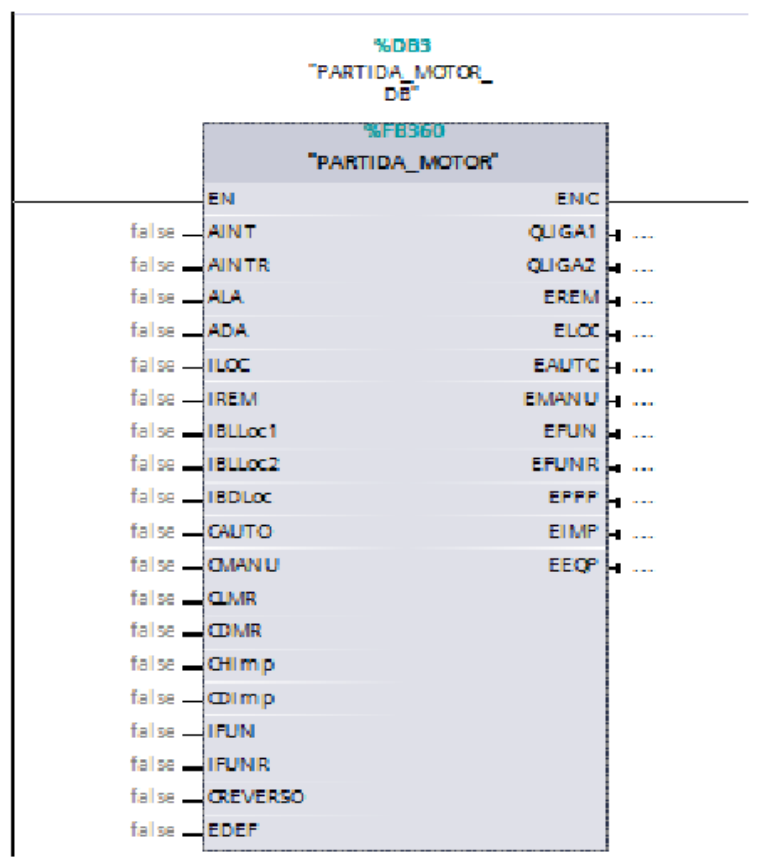

Figura 4. Bloco de Partida de Motor.

Como podemos ver na figura 4, um bloco de partida de motor possui 19 entradas e 11 saídas, entre elas temos estados, defeitos, comandos e intertravamentos. A lógica interna deste bloco foi desenvolvida apenas uma vez e utilizada para a partida de todos os motores da planta apenas indexando a função (FB) criada.

Esta estrutura permitiu um software bem estruturado, reduzindo a ocorrência de desvios de lógica no funcionamento dos equipamentos, e de integração com o sistema supervisório.

Para atender a segunda condição, podemos verificar que o PLC 1A e PLC 6 estavam diretamente interligados ao processo controlado pelo PLC 1. Nesta condição, qualquer falha interna ou na interface entre estes PLCs parariam todo o processo. E o restabelecimento do processo poderia necessitar de atuação em mais de um PLC. Para aumentar a robustez e facilitar a atuação da manutenção, os racks dos PLCs $1 \mathrm{~A}$ e 6 foram transformados em remotas do PLC 1 e suas respectivas lógicas integradas, eliminando as interfaces via $\mathrm{l} / \mathrm{O}$.

No caso do PLC 02 aconteceu uma situação contrária e uma semelhante a do PLC 1. Os $\mathrm{I} / \mathrm{Os}$ do resfriador da Sinterização foram para um PLC exclusivo, denominado PLC 9, onde houve necessidade da criação de uma nova interface de intertravamento. Enquanto que o PLC 3, cujos sinais em sua grande maioria (95\%) correspondiam ao processo do PLC 2, foi transformado em remota do PLC 2, e sua lógica incorporada.

Desta forma a nova configuração de PLCs da Sinterização é mostrada na figura 5.

* Contribuição técnica ao $18^{\circ}$ Seminário de Automação e TI Industrial, 23 a 26 de setembro de 2014, São Paulo, SP, Brasil. 

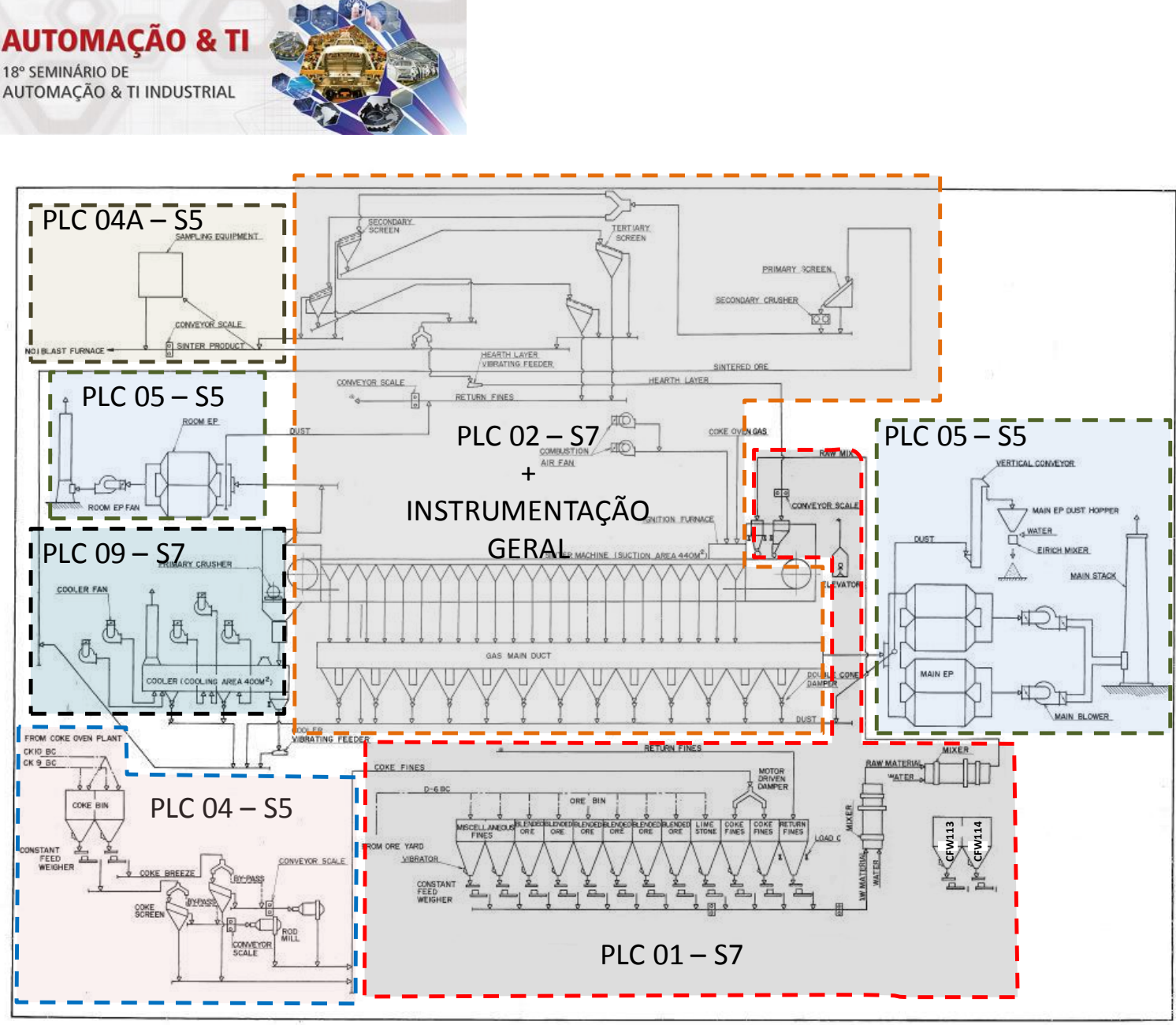

Figura 5. Configuração dos PLCs da Sinterização em 2012.

O desenvolvimento do projeto e a migração atenderam aos prazos definidos originalmente sem ocorrência de desvios críticos dentro do que foi proposto. A interface com nível 02 foi adequada e reestabelecida durante a operação assistida.

\section{$2.22^{\text {a }}$ Etapa de Migração - 2014}

A segunda etapa de migração, o desafio era conseguir realizar uma migração sem a necessidade de uma grande parada.

Nesta etapa os PLCs 4 e 4A podem ser migrados individualmente, pois não existe interface entre eles, o que é uma vantagem. Porém após a experiência adquirida na $1^{\text {a }}$ etapa, concluímos que não seria viável substituir os painéis e todo o hardware, pois somente para esta atividade seria necessário no mínimo 03 dias, levando a migração total de cada um para pelo menos 07 dias, o que seria inviável operacionalmente.

Foi pesquisada outra solução no manual de migração do PLC S5 disponível no site da Siemens [3], e verificado que o hardware do PLC S5 instalado nos PLCs 4 e 4A permitiam o reaproveitamento dos I/Os S5 usando uma placa de interface com 0 PLC S7. Para utilizar esta solução seria necessário utilizar um rack principal da família S7-400, por limitação da solução Siemens.

Escolhemos o PLC 4A da Amostragem de Sinter porque ele não interfere diretamente no processo produtivo, possui menor quantidade de $\mathrm{l} / \mathrm{O}$ e o rack principal não possui nenhum I/O instalado.

Utilizamos o mesmo padrão de software desenvolvido na $1^{\text {a }}$ etapa, pois os I/Os do S5 eram mapeados como variáveis internas de memória do novo PLC.

As atividades da migração foram a desmontagem do rack principal S5, instalação do novo rack S7, instalação do cartão de interface na $1^{\text {a }}$ remota do PLC S5, e

* Contribuição técnica ao $18^{\circ}$ Seminário de Automação e TI Industrial, 23 a 26 de setembro de 2014, São Paulo, SP, Brasil. 
interligação entre a remota e o rack principal. Todas estas foram realizadas no período de $03 \mathrm{~h}$. Uma vez a comunicação entre os racks funcionando, foi possível partir para os testes (bit a bit, testes a frio, testes com carga e start-up). Todo processo levou apenas 03 dias, quando foi iniciada a operação assistida da Amostragem.

\section{RESULTADOS E DISCUSSÃO}

Avaliando as duas etapas de migração, podemos verificar os seguintes fatos.

Na primeira etapa:

- Substituição total do hardware, incluindo painéis;

- Etapa de montagem com mais de 10 dias de duração, incluindo os testes bit a bit;

- Mudanças na configuração dos PLCs existentes, transformação de 03 PLCs em remotas;

- Adequação e Unificação do Sistema Supervisório, adaptado a nova estrutura de software dos PLCs;

Na segunda etapa:

- Substituição mínima do hardware, sem intervenção em cabos de I/O;

- Etapa de montagem de poucas horas de duração;

- Preservação da mesma configuração dos PLCs, pois como o PLC 4 e 4A se localizam fisicamente em locais distintos, não seria possível torna-los apenas um PLC como era originalmente;

- Ainda não foi concluída, restando a substituição do PLC 4 (hardware fornecido, software desenvolvido e parcialmente testado);

As duas etapas possuíam condições de implantação distintas, onde na primeira havia necessidades além da simples substituição dos PLCs como a melhoria na seletividade de controle e a unificação do Sistema Supervisório. As soluções implementadas em cada uma delas mostrou-se a mais adequada as suas respectivas condições, atendendo plenamente a necessidades particulares de cada processo.

\section{CONCLUSÃO}

Concluímos que a utilização de soluções distintas, cada uma delas se adequando as condições de trabalho, principalmente em relação ao prazo de execução que normalmente é o fator decisivo neste tipo de serviço, ambas podem ser utilizada com sucesso. No caso da Sinterização da AMT, é importante ressaltar que a sequencia que cada solução foi implantada foi favorável, pois na $2^{\mathrm{a}}$ etapa toda estrutura de software já havia sido consolidada, e o hardware do PLC S5 desativado na $1^{\text {a }}$ etapa serve de sobressalente, suportando novas migrações aproveitando os I/Os originais, como realizada na $2^{a}$ etapa.

\section{Agradecimentos}

Todas as pessoas que colaboraram nas diferentes etapas do projeto: avaliação, aprovação, contratação, engenharia, montagem, start-up, operação assistida.

A empresa TSA que desenvolveu e executou este projeto sob a orientação da AMT.

* Contribuição técnica ao $18^{\circ}$ Seminário de Automação e TI Industrial, 23 a 26 de setembro de 2014, São Paulo, SP, Brasil. 


\section{REFERÊNCIAS}

1 Instituto Aço Brasil, 2009. O Aço - Processo Siderúrgico. [online] Disponível em: $<$ http://www.acobrasil.org.br/site/portugues/aco/processo--etapas.asp> [Acessado em 1 de Maio de 2014].

2 Starling AR. Avaliação de metodologias de simulação física do processo de sinterização. Dissertação de Mestrado. Curso de Pós-graduação em Engenharia Metalúrgica e de Minas. UFMG, 2012

3 Brisky R. SIMATIC S5 to S7 Migration. Março 2006, Atualizado em Junho 2007 [acesso em 26 ago. 2013];: Disponível em: http://www.siemens.com 